\title{
E-Periodica: die Plattform für digitalisierte Schweizer Zeitschriften
}

\begin{abstract}
E-Periodica ist eine von der ETH-Bibliothek betriebene Open AccessPlattform für digitalisierte Schweizer Zeitschriften aus verschiedenen wissenschaftlichen Bereichen und zu kulturellen und sozialpolitischen Themen. Die aufgeschalteten Bestände erstrecken sich vom 18. Jahrhundert bis zu den jeweils neuesten Jahrgängen. Im Rahmen von E-Periodica bestehen verschiedene Kooperationen mit Schweizer Bibliotheken und weiteren Partnern, die den Betrieb und Ausbau des Angebotes aktiv unterstützen. Im Verlauf des mehrjährigen Bestehens der Plattform wurden verschiedene Massnahmen getroffen, um deren langfristigen Betrieb gewährleisten zu können. Im vorliegenden Beitrag wird E-Periodica anhand finanzieller, rechtlicher sowie organisatorischer Aspekte vorgestellt und es werden die zur Aufschaltung einer Zeitschrift erforderlichen Arbeitsprozesse beschrieben.
\end{abstract}

\section{Einleitung}

E-Periodica ist eine von der ETH-Bibliothek ${ }^{1}$ betriebene Open Access-Plattform für digitalisierte Zeitschriften und periodisch erscheinende Publikationen aus der Schweiz oder mit Bezug zur Schweiz. Inhaltlich erstreckt sich das Angebot über Naturwissenschaften, Geschichte, Kunst und Kultur bis hin zu sozialwissenschaftlichen Themen und Religionswissenschaft. Zur Verfügung gestellt werden die gesamten Retrobestände der Zeitschriften. Oft befinden sich darunter auch längst vergriffene Ausgaben oder Jahrgänge aus Rara-Beständen, die nicht oder nur schwer zugänglich sind. Die ältesten Jahrgänge der auf E-Periodica aufgeschalteten Zeitschriften gehen zurück bis ins 18. Jahrhundert. Zusätzlich zu den retrodigitalisierten Beständen werden bei vielen noch laufenden Zeitschriften jeweils auch die neuen Hefte respektive Jahrgänge aufgeschaltet. ${ }^{2}$

Die Inhalte stehen als Volltexte zur Verfügung und können online gelesen oder als PDF heruntergeladen werden. Dabei werden keine Nutzungsgebühren

1 https://www.library.ethz.ch/de/. Alle Links in diesem Beitrag wurden am 26.03.2018 überprüft.

2 Stand Juli 2017: 267 Zeitschriften mit insgesamt rund 6 Mio. Seiten online.

Ә Open Access. (C) Regina Wanger, publiziert von De Gruyter. (c) BY-NC-ND Dieses Werk ist lizenziert unter der Creative Commons Attribution-NonCommercial-NoDerivatives 4.0 Lizenz. 
erhoben. Je nach Vertrag mit den jeweiligen Rechteinhabern können jedoch die Volltexte neuerer Ausgaben bis zu maximal fünf Jahren gesperrt bleiben.

Genutzt wird E-Periodica von Studierenden und Forschenden, aber auch von Personen, die aus beruflichen oder persönlichen Gründen auf Informationssuche sind. Und nicht zuletzt sind es auch Abonnentinnen und Abonnenten, die von einer zusätzlichen digitalen Verfügbarkeit ihrer Zeitschrift profitieren. Der Nutzerkreis lässt sich somit sehr breit abstecken.

Im Folgenden wird E-Periodica in Form eines Praxisberichtes vorgestellt. Dabei werden die Entstehung der Plattform sowie organisatorische, finanzielle und technische Aspekte beschrieben und es wird ein kurzer Ausblick in die zukünftige Entwicklung gewährt.

\section{Geschichte}

Das Konzept für E-Periodica entstand im Rahmen des gesamtschweizerischen Innovations- und Kooperationsprojektes e-lib.ch: Elektronische Bibliothek Schweiz ${ }^{3}$, unter dessen Dach Dienstleistungen und Instrumente für die wissenschaftliche Arbeit aufbereitet und angeboten wurden. Die Aufschaltung der Plattform erfolgte damals mit der Bezeichnung „retro.seals.ch“ (seals: swiss electronic academic library service). $\mathrm{Zu}$ Beginn standen bei retro.seals.ch ausschliesslich wissenschaftliche Publikationen im Fokus. Die ersten aufgeschalteten Zeitschriften waren denn auch mathematische Publikationen. Bald folgte das sogenannte ,Baugedächtnis' mit Zeitschriften zum Thema Architektur in der Schweiz. Nach und nach kamen Zeitschriften zu weiteren Themen dazu und schliesslich wurden die Aufnahmekriterien etwas gelockert, indem nicht mehr nur rein wissenschaftliche Publikationen aufgenommen wurden. Die Federführung bei retro.seals.ch hatte zu Beginn das Konsortium der Schweizer Hochschulbibliotheken. ${ }^{4}$ Mit Abschluss des Gesamtprojektes e-lib.ch im Jahr 2013 übernahm die ETH-Bibliothek die alleinige Verantwortung für retro.seals.ch.

Das Interesse an der Plattform war gross, auch von Seiten der Herausgeber, so dass das Zeitschriftenangebot kontinuierlich ausgebaut werden konnte. Im ursprünglichen Konzept von retro.seals.ch war jedoch keine derart grosse Anzahl Zeitschriften vorgesehen und allmählich liessen sich die Verwaltung des stetig steigenden Datenvolumens sowie die verschiedenen Arbeitsprozesse nicht mehr mit der nötigen Effizienz bewältigen. Dies erforderte schliesslich eine umfassende

3 http://www.e-lib.ch/.

4 http://www.consortium.ch/. 
Neukonzeption der vorhandenen technischen Infrastruktur. In Zusammenarbeit mit der Betreiberfirma der Software entwickelte das zuständige Team des IT-Services der ETH-Bibliothek eine massgeschneiderte Lösung für die Plattform. Im Rahmen dieser Entwicklung wurde auch der Webauftritt einem Redesign unterzogen und das Angebot umbenannt in „E-Periodica“. Der Launch der neuen Webseite fand im März 2016 statt.

\section{Organisation}

Die Organisation von E-Periodica wird hier anhand der verschiedenen involvierten Parteien sowie weiterer Komponenten beschrieben.

Gesamtverantwortung ETH-Bibliothek

Leitung und Organisation von E-Periodica sind im DigiCenter ${ }^{5}$ der ETH-Bibliothek angesiedelt. Die Arbeiten im Bereich Digitalisierung, Qualitätskontrolle und Strukturierung der Daten werden vorwiegend durch studentische Mitarbeitende des DigiCenters durchgeführt. Der Betrieb der IT-Infrastruktur der Plattform sowie die Speicherung der Daten erfolgen an der ETH-Bibliothek respektive an der ETH Zürich. Die Betreuung der Scan-Infrastruktur wird durch Mitarbeitende des IT-Services der ETH-Bibliothek gewährleistet.

Kooperation mit der Schweizerischen Nationalbibliothek

Die Schweizerische Nationalbibliothek $(\mathrm{NB})^{6}$ ist seit 2009 Kooperationspartnerin von E-Periodica und nutzt die Plattform im Rahmen ihres gesetzlichen Auftrags, Helvetica zu vermitteln. Dazu wählt die NB Zeitschriften aus ihren eigenen Beständen für die Aufschaltung aus, wobei diese Auswahl häufig mehrere Publikationen zu einem bestimmten Themenkreis umfasst. Dabei ist die NB selbst zuständig für die Digitalisierung ihrer Zeitschriften sowie die Strukturierung der Daten. Für Letzteres verfügt die NB über einen Remote-Zugang direkt auf den XML-Editor der ETH-Bibliothek (vgl. Abschnitt „Strukturierung“ unten). Die vollständig aufbereiteten Daten werden schliesslich von der ETH-Bibliothek in E-Periodica importiert.

5 https://www.library.ethz.ch/de/ms/DigiCenter.

6 http://www.nb.admin.ch/. 


\section{Vertragspartner}

Die Aufnahme einer neuen Zeitschrift in E-Periodica wird mit den involvierten Parteien - dies sind in der Regel Rechteinhaber, Herausgeber, Redaktionen (im Folgenden als ,Vertragspartner' bezeichnet) - anhand eines schriftlichen Vertrages geregelt. Ausnahmen werden diesbezüglich nur bei sogenannten ,verwaisten', insbesondere älteren Publikationen gemacht, bei denen keine Rechteinhaber mehr ermittelt werden können.

Organisatorische und finanzielle Beteiligung Dritter

Oft werden die Vertragspartner organisatorisch und/oder finanziell unterstützt durch Dritte. So fördert beispielsweise die Schweizerische Akademie der Geistesund Sozialwissenschaften (SAGW) ihre Mitglieder aktiv bei der Aufschaltung der eigenen Publikationen und beteiligt sich an den Kosten. Auch verschiedene Schweizer Bibliotheken unterstützen Zeitschriftenprojekte, indem sie Zeitschriften aus ihren eigenen Beständen zur Verfügung stellen, Nutzungsrechte abklären sowie Aufbereitung und Betrieb mitfinanzieren. Es sind mittlerweile verschiedene Bibliotheken mit oftmals mehreren Publikationen bei E-Periodica vertreten - zum Beispiel die Universitätsbibliothek Bern, die Bibliothek am Guisanplatz in Bern, die Kantonsbibliothek Thurgau in Frauenfeld oder die Kantonsbibliothek Appenzell Ausserrhoden in Trogen AR.

\section{Aufnahmekriterien}

Während die Plattform zu Beginn auf wissenschaftliche Zeitschriften fokussiert war, wurde diese Beschränkung allmählich zugunsten eines breiteren Angebotes gelockert. Entscheidungsgrundlage für die Aufnahme einer neuen Zeitschrift bilden nun unterschiedliche Kriterien. Die folgenden Kriterien sind zwingend:

- ein zeitschriftenartiger Charakter (regelmässige Erscheinungsweise)

- das Einverständnis der Rechteinhaber (sofern es sich nicht um eine verwaiste Publikation handelt)

- der Bezug zur Schweiz (z.B. auch thematisch, Sitz des Herausgebers etc.)

Zusätzlich gibt es weitere Kriterien, die nicht unbedingt und vollumfänglich erfüllt sein müssen. Sie dienen eher als Richtlinien und werden individuell gewichtet:

- nicht-kommerzieller Charakter

- kein gleichwertiges Online-Angebot an anderer Stelle

- Grundlagenmaterial für die Forschung

- von allgemeinem Interesse 
Die Ablehnung einer Zeitschrift kann auch erfolgen, wenn ein Risiko besteht, dass der Personen- resp. Datenschutz nicht gewährleistet ist. Davon betroffen sein können etwa Publikationen, die zahlreiche Abbildungen von Privatpersonen mit Namen oder ähnlichen persönlichen Angaben enthalten.

Nachweise, Recherche

Die Zeitschriftenbestände von E-Periodica sind über verschiedenen Kataloge und Datenbanken recherchierbar, zum Beispiel:

- Wissensportal der ETH-Bibliothek: http://www.library.ethz.ch/

- NEBIS-Katalog - Netzwerk von Bibliotheken und Informationsstellen in der Schweiz: http://www.nebis.ch/

- $\quad$ swissbib.ch - Katalog aller Schweizer Hochschulbibliotheken, der Schweizerischen Nationalbibliothek, zahlreicher Kantonsbibliotheken und weiterer Institutionen: https://www.swissbib.ch/

- Elektronische Zeitschriftenbibliothek: http://rzblx1.uni-regensburg.de/ezeit

- EBSCO Information Services: http://www.ebsco.com

Ausserdem führt auch eine Google-Suche zu den Zeitschriften und Volltexten auf E-Periodica.

\section{Rechtliche Regelungen}

Die ETH-Bibliothek besitzt als Betreiberin das Recht zur Nutzung der Zeitschriften ausschliesslich im Rahmen von E-Periodica. Alle anderen Rechte verbleiben bei den bestehenden Rechteinhabern. Mit diesen (und allfälligen weiteren Beteiligten) wird dazu ein schriftlicher Vertrag erstellt. In vielen Fällen ist nicht eindeutig geklärt, ob die Herausgeber der Zeitschrift oder die Autoren und Autorinnen die Rechte an den Artikeln innehaben. Aus Kapazitätsgründen ist es jedoch nicht möglich, die rechtliche Einwilligung aller Urheber einzuholen. Sollte eine Autorin nach Aufschaltung der Publikation die Veröffentlichung auf E-Periodica ablehnen, wird der betreffende Beitrag entfernt und durch einen Platzhalter ersetzt.

Anfragen von Nutzenden zur Verwendung von Inhalten werden jeweils den Rechteinhabern weitergeleitet. Erst mit deren Einwilligung wird auf Wunsch ein Masterfile zur Weiterverwendung an den Nutzer herausgegeben.

Angaben zum Nutzungsrecht finden sich auf der Plattform. Zudem wird bei jedem PDF-Download ein Deckblatt generiert, das nebst den wichtigsten Metadaten zum Inhalt auch die allgemeinen Nutzungsrechte enthält.

Finanzierung, Kostenmodell

Anfangs erfolgte die Finanzierung der Plattform durch Projektgelder von e-lib.ch sowie durch finanzielle Beteiligung der Vertragspartner und der ETH-Bibliothek. 
Mit Beendigung von e-lib.ch entfiel der Anteil der Projektgelder und die Finanzierung von E-Periodica basiert seither auf einem Kostenmodell, bestehend aus zwei Kostenarten:

1. Die einmaligen Projektkosten beinhalten die Aufbereitung und Aufschaltung des Retro-Bestandes einer Zeitschrift und die Kosten für den Digital Object Identifier (DOI). Diese werden zu ungefähr zwei Dritteln durch die ETHBibliothek finanziert und ein Drittel wird den Vertragspartnern weiterverrechnet.

2. Die jährlichen Betriebskosten beinhalten die Aufbereitung und Aufschaltung der neuen Jahrgänge (sofern vereinbart) sowie das Datenhosting resp. die Langzeitarchivierung der Daten. Diese Kosten sind beträchtlich geringer als die einmaligen Projektkosten und werden den Vertragspartnern vollumfänglich weiterverrechnet.

Das Kostenmodell soll eine übermässige finanzielle Belastung der Vertragspartner verhindern und dadurch deren Teilnahme an E-Periodica erleichtern oder überhaupt erst ermöglichen. Die ETH-Bibliothek ihrerseits profitiert durch ihre finanzielle Beteiligung von einem breiteren Zeitschriftenangebot, was sich wiederum positiv auf die Nutzungszahlen und Präsenz der Plattform auswirkt.

\section{Prozesse - Workflow}

Im Folgenden werden die verschiedenen Arbeitsschritte von den ersten Abklärungen bis zur Aufschaltung und zur Datenarchivierung einer neuen Zeitschrift auf E-Periodica erläutert.

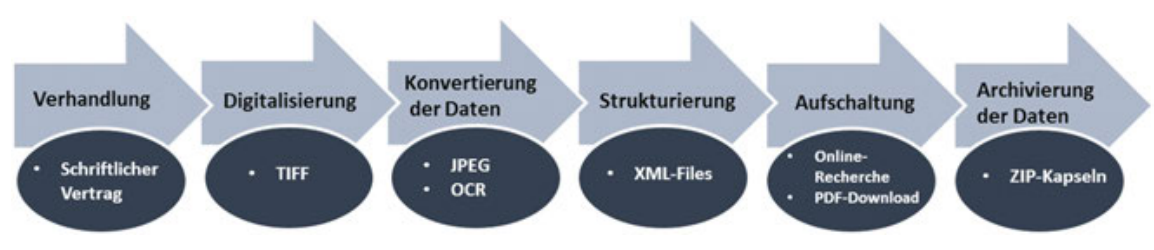

Abb. 1: Prozess Zeitschriftenprojekt E-Periodica

Abklärung und Verhandlung

Der erste Impuls zur Aufnahme einer neuen Zeitschrift in E-Periodica erfolgt in den meisten Fällen durch die Herausgeber oder Redaktionsmitglieder. Möglich ist aber auch der umgekehrte Weg, indem die ETH-Bibliothek mit einer Anfrage an 
die Verantwortlichen einer Zeitschrift herantritt. Dies ist beispielsweise dann der Fall, wenn ein bestehender Themenkreis ergänzt werden soll oder auch eine Anregung einer Nutzerin oder eines Nutzers weiterverfolgt wird.

Besteht von allen Beteiligten (insbesondere von den Rechteinhabern) ein grundsätzliches Einverständnis zur Aufnahme der Zeitschrift, werden die rechtlichen, finanziellen und organisatorischen Belange geklärt und in einem schriftlichen Vertrag festgehalten. ${ }^{7}$ Der Inhalt des Vertrags ist grundsätzlich standardisiert. Aus organisatorischen und verwaltungstechnischen Gründen sind individuelle Abmachungen nur in sehr beschränktem Masse möglich.

\section{Digitalisierung}

Meist stellen die Vertragspartner einen Bestand der Zeitschrift für die Digitalisierung zur Verfügung. Sofern es sich dabei um einen nicht mehr benötigten Doublettenbestand handelt, wird dieser aufgeschnitten und im Anschluss an die Digitalisierung entsorgt. Andernfalls wird der Bestand nach Abschluss sämtlicher Arbeiten wieder unversehrt zurückgegeben. Es werden zum Teil auch Bestände der ETH-Bibliothek für die Digitalisierung verwendet. Sollte die Zeitschrift bereits durch Dritte digitalisiert vorliegen und die Qualität der Dateien den Standards von E-Periodica entsprechen, können die bestehenden Files übernommen werden.

Die Zeitschriften werden im Format TIFF mit einer Auflösung von 300dpi (je nach Vorlage in Farbe oder Graustufe) gescannt. Die Digitalisierung umfasst die gesamte Zeitschrift inklusive Werbung, Rubriken, Vereinsnachrichten etc. Im Anschluss an die Digitalisierung erfolgt eine standardisierte Qualitätskontrolle, bei der die digitalen Daten auf Vollständigkeit und Bild- und Farbqualität geprüft werden. Mängel, beispielsweise bezüglich Farbqualität oder Schriftschärfe, können mit der automatisierten Bildbearbeitung behoben werden.

Datenkonvertierung

Im Rahmen der oben erwähnten Neukonzeption der IT-Infrastruktur wurde ein sogenannter ,Workflow-Client' entwickelt, der eine zentrale Steuerung der Prozesse und Verwaltung der Daten ermöglicht. Die nachfolgend beschriebenen Prozessschritte lassen sich damit erheblich effizienter und einfacher gestalten.

Die neu zu erschliessende Zeitschrift wird im Workflow-Client mit den zentralen Metadaten (zum Beispiel Titel der Zeitschrift, Herausgeber, Erscheinungsjahre) erfasst. Anschliessend werden die für den Import bereitliegenden TIFFMasterfiles in den Workflow-Client geladen. Dies wiederum löst im Hintergrund weitere Prozesse aus:

7 Im Juli 2017 bestehen im Rahmen von E-Periodica 140 Verträge. 
- Es werden JPEG-Derivate unterschiedlicher Auflösung aus den TIFF-Files generiert, die für die Strukturierung im XML-Editor, die Online-Präsentation, die Zoomfunktion und den PDF-Download benötigt werden.

- Parallel zum Laden der TIFF-Files wird auch der OCR-Server angestossen, der aus diesen Files Volltextdateien erstellt. Je nach Konfiguration werden Antiqua-, Fraktur- oder gemischte OCR-Dateien generiert.

\section{Strukturierung}

Im Anschluss an die Datenkonvertierung erfolgt die Strukturierung der Zeitschrift in einem XML-Editor, der ebenfalls Bestandteil des Workflow-Client ist:

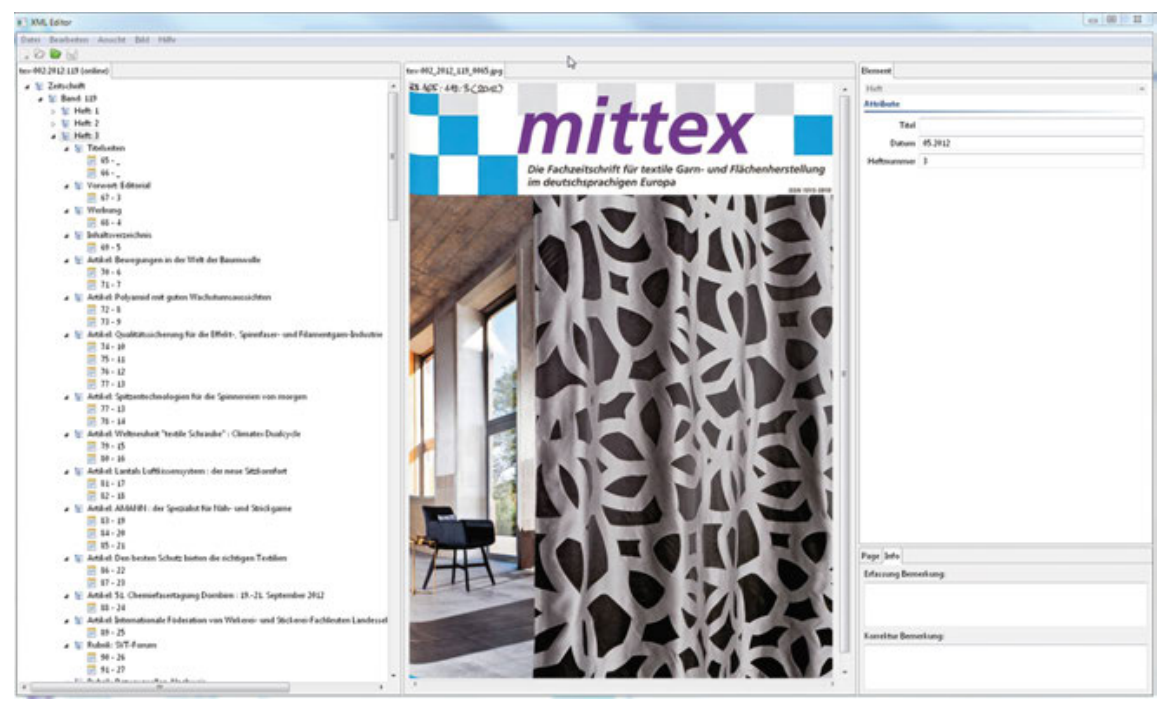

Abb. 2: XML-Editor: Erfassen von Strukturmetadaten

Im linken Bereich des XML-Editors ist die Baumstruktur des Inhalts mit den bereits erfassten Strukturmetadaten ersichtlich. Im mittleren Abschnitt wird das jeweilige JPEG der links selektierten Seite angezeigt. Im rechten Bereich werden die Strukturmetadaten manuell erfasst. Diese beinhalten unter anderem:

- die Eingabe von Autoren und Artikeltiteln

- die Paginierung

- die Kennzeichnung der Art des Inhaltes durch sogenannte ,Elemente‘ wie zum Beispiel Artikel, Vorwort, Rubrik, Nachruf, Werbung etc.

- die Verknüpfung von Anhängen (beispielsweise Tabellen oder Tafeln) mit dem zugehörigen Artikel 
Der Zeitaufwand für die manuelle Strukturierung kann je nach Inhalt und Struktur einer Zeitschrift beträchtlich variieren und ist insbesondere bei umfangreichen Publikationen nur schwer planbar. Auch müssen gelegentlich Kompromisse gefunden werden, da nicht jede Spezialität einer Zeitschrift abgebildet werden kann.

\section{Aufschaltung}

Vor der definitiven Aufschaltung wird die Zeitschrift zuerst in einer Testumgebung mittels standardisierten Kontrollfragen $\mathrm{zu}$ verschiedenen Ansichten und Funktionen geprüft. Nach Behebung allfälliger Fehler wird die Zeitschrift aufgeschaltet und steht dann auf E-Periodica zur Verfügung.

Über Neuaufschaltungen wird jeweils auf ETH-News-Kanälen und über Mailinglisten informiert. Meist erfolgt auch eine Ankündigung der Vertragspartner selbst, die auf ihren Webseiten, mittels Medienmitteilung oder in einer aktuellen Ausgabe der Zeitschrift über die Aufschaltung in E-Periodica berichten.

\section{Erstellung von Archivkapseln}

In einem letzten Schritt werden Archivkapseln generiert. Für jeden abgeschlossenen Zeitschriftenjahrgang wird eine unkomprimierte ZIP-Datei aus den originalen TIFF-Dateien, den OCR-Dateien und dem Struktur-XML erstellt. Müssen nachträglich Korrekturen an einem Zeitschriftenjahrgang vorgenommen werden seien dies Modifikationen in den Strukturmetadaten, den bibliographischen Metadaten oder in den Bildern und OCR-Dateien - wird eine Delta-Archivkapsel generiert, die ausschliesslich den geänderten Datensatz enthält. Die ursprüngliche Archivkapsel wird im Falle einer nachträglichen Korrektur nicht ersetzt oder geändert.

\section{Webauftritt}

Der Webauftritt der Plattform wurde im Jahr 2016 komplett umgestaltet. Das neue, barrierefreie Design ist responsive, das heisst, die Darstellung der Webseite wird der verwendeten Bildschirmgrösse angepasst (Desktop, Tablet, Smartphone). Auf der Einstiegsseite wird jede Zeitschrift mit ihrem Cover präsentiert, was zugleich die breite Palette und Vielfalt des Zeitschriftenangebotes visualisieren soll. Im Rahmen des Redesigns wurden zudem Recherchefunktionen und Anzeigemöglichkeiten optimiert. Der neue Webauftritt wurde beim Best of Swiss Web Award $2016^{8}$ mit drei Medaillen ausgezeichnet (Bronze in der Kategorie ,Mobile Web` und Silber in den Kategorien ,Usability“ und ,Technology‘). 
4. digitalisierte zeitschriften ETHBMBLTHEK

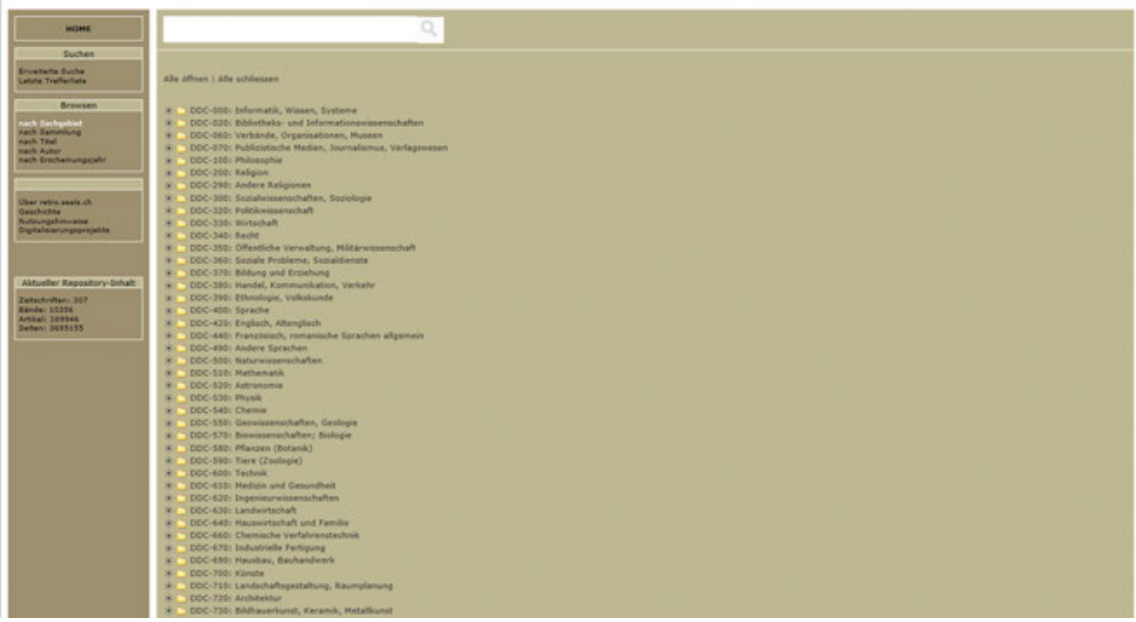

Abb. 3: Homepage retro.seals.ch (bis 2015)

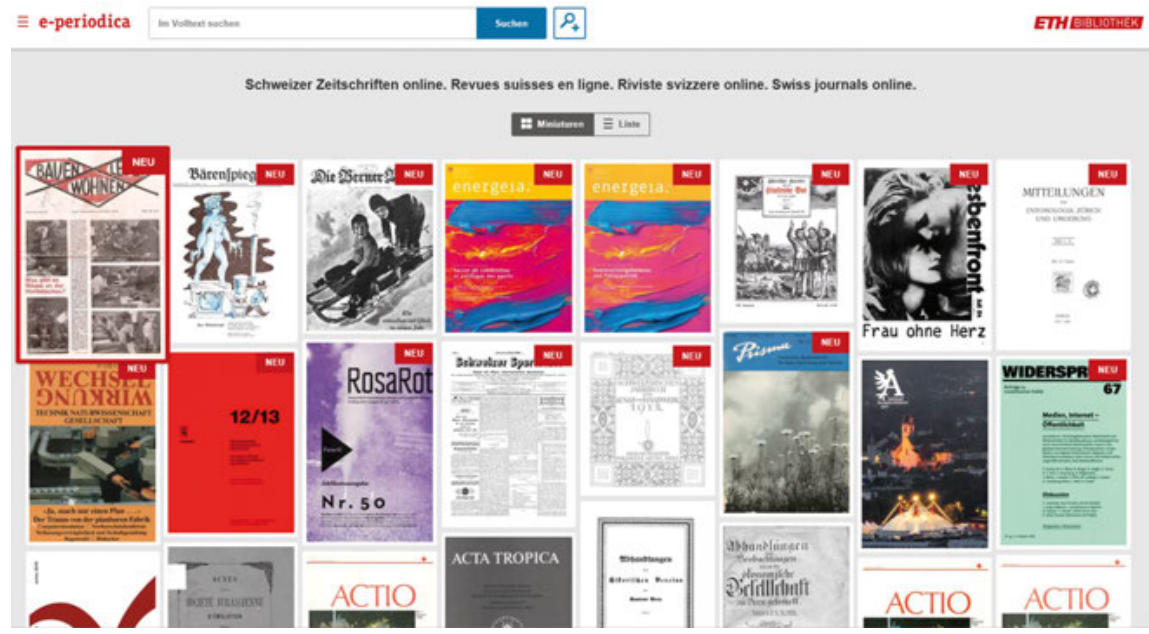

Abb. 4: Homepage E-Periodica (ab 2016)

8 http://www.bestofswissweb.ch/. 
Inhalte nutzen und teilen

Auf der Plattform stehen verschiedene Recherche- und Anzeigemöglichkeiten sowie eine Zoomfunktion zur Verfügung. Besonders zu erwähnen ist der sogenannte ,Split-View': Je nach Recherchemodus wird entweder auf der linken Seite die Trefferliste oder das im Vorfeld manuell strukturierte Inhaltsverzeichnis angezeigt, während rechts eine Seitenvorschau eingeblendet wird.

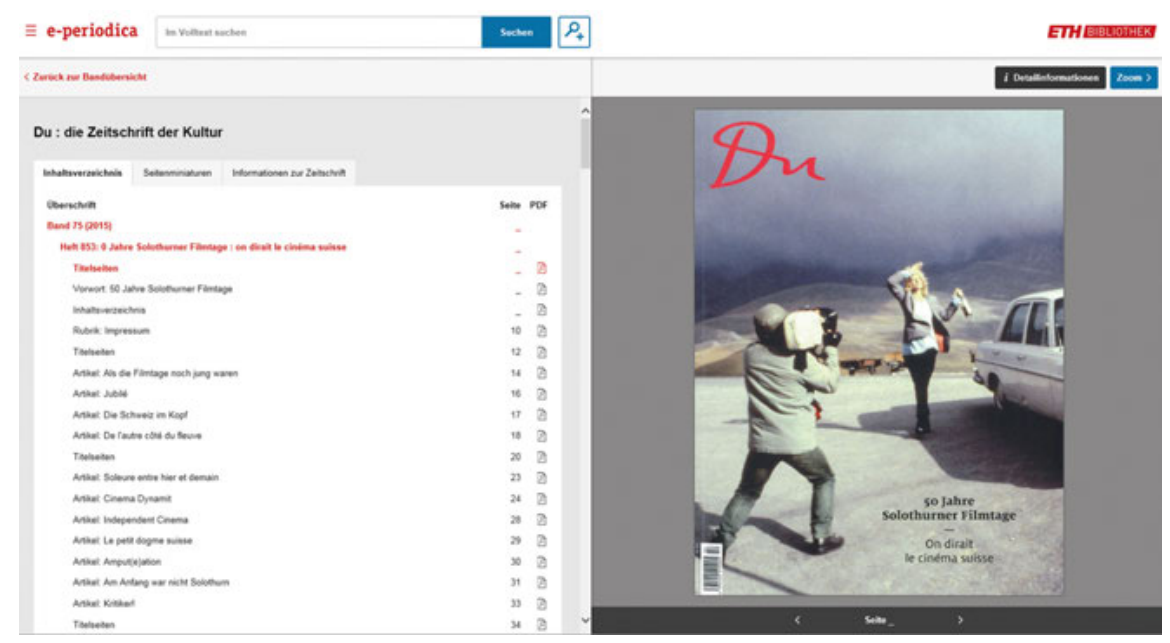

Abb. 5: Split-View mit Inhaltsverzeichnis und Seitenansicht

PDF-Download: Die Volltexte können als PDF heruntergeladen werden. Das PDF wird dabei jeweils direkt beim Download aus den vorhandenen JPEG-Dateien erstellt. Aus Speicherplatzgründen sind auf der Plattform keine PDF-Dateien hinterlegt. Beim Download wird jeweils ein Deckblatt mit den wichtigsten Metadaten zum betreffenden Inhalt sowie den Nutzungsbestimmungen generiert und als erste Seite direkt in das PDF eingefügt.

Digital Object Identifier (DOI): Die bei der Strukturierung als „Artikel“ gekennzeichneten Inhalte werden zur dauerhaften Auffindbarkeit mit einem Digital Object Identifier versehen. Registriert werden diese über den DOI-Desk der ETH Zürich. ${ }^{9}$

Social Media: Die Artikel können über Twitter, Facebook, Google+ und Pinterest geteilt werden.

9 DOI-Desk, ETH Zürich: https://www.library.ethz.ch/de/Dienstleistungen/Publizieren-registrie ren-verwalten/DOI-Desk-der-ETH-Zuerich. 


\section{Sperrfrist}

Neue Hefte oder Jahrgänge einer Zeitschrift können auf Wunsch der Vertragspartner bis zu maximal fünf Jahren gesperrt werden. Diese gesperrten Ausgaben werden in E-Periodica bereits aufgeschaltet, wobei das Browsen im Inhaltsverzeichnis möglich ist. Der Zugriff auf den Volltext wird hingegen nicht freigeschaltet. Gesperrte Ausgaben sind jeweils mit einem Schloss-Icon und einem entsprechenden Hinweis gekennzeichnet. Nach Ablauf der Sperrfrist werden die Volltexte automatisch freigegeben.

\section{Fazit und Ausblick}

E-Periodica hat sich als Plattform im Bereich Zeitschriftendigitalisierung etabliert. Aufgrund des steten Ausbaus mit neuen Zeitschriften und der laufenden thematischen Erweiterung geniesst das Angebot eine hohe Aufmerksamkeit und Akzep$\operatorname{tanz}$ - Zugriffszahlen und Rückmeldungen lassen darauf schliessen.

$\mathrm{Zu}$ diesem Erfolg beigetragen haben schliesslich verschiedene Faktoren. Dazu gehören selbstverständlich das Interesse und die Bereitschaft der Herausgeber und Redaktionen, ihre Zeitschrift auf E-Periodica zur Verfügung zu stellen, sowie das Engagement der Kooperationspartner im organisatorischen und finanziellen Bereich. In Kombination mit dem von der ETH-Bibliothek mitgetragenen Kostenmodell konnte und kann die Aufnahme einer Vielfalt an Publikationen überhaupt erst ermöglicht werden. Des Weiteren macht sich ein gesteigertes Bewusstsein in Bezug auf Open Access bemerkbar. Die Vertragspartner schätzen es, kein eigenes Open Access-Angebot entwerfen zu müssen, sondern die vorhandene Infrastruktur und entsprechende Synergien nutzen zu können. Und es ist auch gelungen, die Plattform im Verlauf des mehrjährigen Bestehens geänderten Voraussetzungen und (Nutzer-)Bedürfnissen anzupassen.

E-Periodica wird weiterhin neue Zeitschriften aufnehmen. Entsprechende Anfragen und Verhandlungen laufen derzeit bezüglich verschiedener Publikationen.

Abgesehen vom eigentlichen Zweck der Plattform - den Zugang zu digitalisierten Zeitschriften anzubieten - verfügt die ETH-Bibliothek mit diesem Bestand auch über eine enorme Menge an qualitativ hochwertigen und gut strukturierten Daten, aus denen gegebenenfalls ein weiterer Nutzen generiert werden kann. Konkret werden derzeit Möglichkeiten im Bereich Text Data-Mining geprüft: In einer Forschungskooperation mit dem Institut für Computerlinguistik der Universität Zürich ist ein Pilotprojekt im Gang, in dessen Rahmen anhand von computerlinguistischen Verfahren automatisierte Korrekturen von OCR-Fehlern sowie die Erkennung und Verlinkung von Eigennamen durchgeführt werden. Basis für das 
Verfahren bildet ein begrenzter Datenbestand von E-Periodica. Ziel dieses Pilotprojektes ist es zu prüfen, ob und inwiefern in dieser Form aufbereitete Inhalte der Forschung als Textkorpora zur Verfügung gestellt werden können. 